\title{
Reflets
}

Revue ontaroise d'intervention sociale et communautaire

\section{Jardins communautaires et mobilisation sociale à Cornwall - Entrevue avec Marc Bisson}

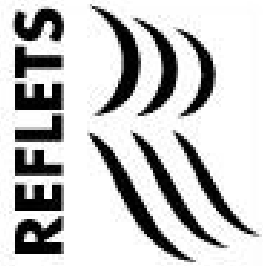

\section{Nérée St-Amand}

Volume 6, numéro 2, automne 2000

Problèmes sociaux en Ontario français

URI : https://id.erudit.org/iderudit/026323ar

DOI : https://doi.org/10.7202/026323ar

Aller au sommaire du numéro

Éditeur(s)

Reflets : Revue ontaroise d'intervention sociale et communautaire

ISSN

1203-4576 (imprimé)

1712-8498 (numérique)

Découvrir la revue

Citer ce document

St-Amand, N. (2000). Jardins communautaires et mobilisation sociale à

Cornwall - Entrevue avec Marc Bisson. Reflets, 6(2), 203-216.

https://doi.org/10.7202/026323ar

Tous droits réservés (C) Reflets : Revue ontaroise d'intervention sociale et communautaire, 2000
Ce document est protégé par la loi sur le droit d'auteur. L'utilisation des services d'Érudit (y compris la reproduction) est assujettie à sa politique d'utilisation que vous pouvez consulter en ligne.

https://apropos.erudit.org/fr/usagers/politique-dutilisation/ 


\section{Jardins communautaires et mobilisation sociale à Cornwall}

Entrevue avec Marc Bisson, promoteur-santé du Centre de santé communautaire de l'Estrie

N.St-Amand : Pourriez-vous, dire un peu qui vous êtes, le genre de travail que vous faites?

M. Bisson : Je suis promoteur-santé dans un centre de santé communautaire de la région de Cornwall et des régions rurales de Crysler et d'Alexandria, dans les comtés de Stormont, Dundas et Glengarry. Depuis 1993, j'oeuvre au sein du Centre de santé communautaire de l'Estrie, d'abord à titre d'agent de développement communautaire et depuis deux ans, comme promoteursanté.

N. St-Amand : De 1993 à 1998, à peu près, vous avez été organisateur communautaire?

M. Bisson : Exactement, et c'est au cours de cette période-là que nous avons développé le Programme de jardin communautaire dont je vais parler un peu plus en détail.

N. St-Amand : Alors pouvez-vous nous dire un peu comment a débuté ce projet?

M. Bisson : À l'époque, le Centre de santé a obtenu une petite subvention du ministère de la Santé pour examiner l'insécurité alimentaire dans Stormont, Dundas et Glengarry. Dans cette optique, une diététistechercheure du Centre avait alors entrepris une démarche de recherche assez poussée. Puis elle avait interviewé des acteurs de différents domaines, tels les banques alimentaires, les coopératives alimentaires, pour évaluer plus en détail la situation alimentaire dans certains quartiers pauvres de la région de Cornwall et dans la campagne avoisinante. 
N.St-Amand : Toute la région, donc?

M. Bisson : Oui, mais davantage Cornwall, parce que les personnes interviewées venaient à la banque alimentaire de cet endroit. La recherchiste travaillait donc à partir d'un questionnaire avec des questions telles : "Quel type d'activité aimeriez-vous faire pour sortir du cycle de la pauvreté, ou de manque d'alimentation?" L'idée du jardinage a été mentionnée à quelques reprises, certains mentionnant même l'idée d'un jardin communautaire. Cela devint un des points forts de l'étude. Nous voulions bien poursuivre ce projet. Comme toute bonne agence qui fait une étude, nous avions un objectif en tête : identifier des besoins. Quant aux jardins communautaires, comme organisateur communautaire à l'époque, je m'étais dit : « Ça, c'est un projet extrêmement intéressant auquel travailler, parce qu'il répond à un besoin identifié par la communauté. »

La responsable de la recherche et moi avons donc fait une demande de subvention au Fonds de développement social de l'Ontario, qui avait un programme d'alimentation pour les enfants. On s'est dit qu'il y avait peut-être moyen, avec les enfants, de mettre sur pied des jardins communautaires. Nous nous sommes donc associés au programme de prévention primaire Partir d'un bon pas des écoles francophones de la région de Cornwall et au Comité consultatif $d u$ maire pour un mode de vie sain de la municipalité de Cornwall pour démarrer un programme de jardin communautaire pour les enfants. Nous nous sommes dit : «La clientèle sera relativement facile à aller chercher au niveau de Partir d'un bon pas qui travaillait dans les écoles. La municipalité va nous trouver le terrain et le Centre de santé verra à l'animation et à la gestion de tout ça ".

N. St-Amand : Un projet tripartite, quoi!

M. Bisson : Une espèce de partenariat à trois. Et, à l'époque, on a eu du succès : on a reçu la subvention qu'on avait demandée-25000\$ sur trois ans-une tranche de $10000 \$$ pour la première année, $10000 \$$ pour la deuxième et $5000 \$$ pour la dernière année. Notre premier jardin communautaire voit le jour durant l'été de 1995 avec quatre écoles francophones de la région de Cornwall participant au programme Partir d'un bon pas. Nous nous occupions 
du transport. Nous avons engagé une animatrice pour les jardins, soit une spécialiste du jardinage écologique. Nous nous sommes dit que si les enfants apprennaient à jardiner, il faudrait quand même qu'ils commencent dès le jeune âge à le faire en " compagnonnage ». En tant qu'agence de santé, nous nous préoccupions non seulement de l'aspect nutritionnel, mais aussi des aspects santé, activité physique et autres intérêts.

Dès le premier été, plus de 125 enfants sont allés se promener dans les jardins communautaires, y ont fait pousser des petits pois, des radis et d'autres légumes. Ils sont retournés à l'automne pour voir le résultat de leur récolte. Ce qui nous a frappés à l'époque, c'était de voir les enfants, pendant l'été, venir avec leurs parents arroser leurs petites fleurs, leurs radis, et montrer à leurs parents comment ils étaient fiers de ce petit jardin-là. Et notre objectif, c'était toujours de répondre au besoin identifié au niveau de l'insécurité alimentaire lors de l'étude pour rejoindre les gens de la communauté. Mais là, nous nous sommes dit : «La première étape, c'est d'avoir un terrain, un jardin. Par la suite, ça va être plus facile d'impliquer des gens".

Nous avons donc réussi à vendre l'idée des jardins communautaires à la municipalité grâce aux enfants-c'est difficile d'aller à l'encontre des enfants. Vous savez un peu comment ça marche : c'est beaucoup plus facile de vendre des choses par rapport aux enfants que par rapport aux personnes défavorisées, démunies. Souvent même on nie la présence de ces personnes-là.

N. St-Amand : Si je comprends bien, les enfants étaient les artisans ou les jardiniers, quoi!

M. Bisson : Au départ, oui, avec l'aide des professeurs.Alors nous, au printemps, nous sommes allés avec notre animatrice dans les classes où Partir d'un bon pas intervenait. Avec l'aide de leurs animateurs scolaires, on a organisé des petites sessions avec les enseignantes et enseignants pour partir des semences de radis...

N. St-Amand : ...dans les écoles?

M. Bisson : Oui! Par la suite, les enfants venaient au jardin planter leurs choses, mais avec l'aide des enseignants et de l'animatrice. 
N. St-Amand : Intéressant comme concept.

M. Bisson : Donc, c'est comme ça que nous avons obtenu notre subvention pour le Fonds de développement social. Entre les semences et les récoltes, il y avait quand même plusieurs mois et une animatrice qu'il fallait occuper alors, nous nous sommes dit : «Nous allons combler notre besoin en insécurité alimentaire pendant cette période-là ». Nous nous sommes associés à la banque alimentaire et à d'autres organismes de la communauté pour pouvoir « remplir les trous » des jardins qui n'étaient pas utilisés par les écoles.

La première année, nous n'avions pas beaucoup de jardiniers, à peine quatre ou cinq. Mais pour nous, c'était un succès. Nous allions pouvoir faire la jonction ... nous espérions que ces jardins communautaires, situés quelque part sur un terrain de la municipalité, les écoles les aiment tellement qu'éventuellement, elles les fassent dans leurs propres cours. Notre plan d'action était d'aller chercher les enfants dans une perspective de développement communautaire, de les amener à reproduire dans leur milieu le jardin communautaire et de laisser le terrain libre pour la clientèle première que nous visions, c'est-à-dire, les personnes les plus démunies de la communauté, pour leur permettre de faire du jardinage.

Alors, ces objectifs-là, nous les avons atteints. Mais d'abord, nous nous sommes heurtés à la bureaucratie municipale. Nous avons dû développer des alliances à l'intérieur même de la municipalité pour pouvoir fonctionner.

N.St-Amand : Quel genre d'alliances?

M. Bisson : D'entrer en contact avec les Services environnementaux, puis avec le Service des parcs, et de rencontrer des leaders qui voulaient, eux aussi que des choses changent à l'intérieur de leur municipalité. Alors de fil en aiguille, s'est élargi le partenariat avec la municipalité. De trois personnes autour de la table, nous nous sommes retrouvés à 8 ou 9 et puis 10 pour parler de planification. Eux, ils avaient des intérêts au niveau de l'environnement. Ils sentaient bien que la présence d'enfants dans les parcs pouvait influer sur la municipalité et sur ses politiques environnementales : l'usage des pesticides, le compostage, c'était très, très vaste. Ces gens-là avaient 
des intérêts certains, comme fonctionnaires, à vouloir que ça se développe. Par ailleurs, il y avait certains blocages bureaucratiques. Mais sur le terrain, ces gens-là avaient intérêt à ce que ça fonctionne.

Par cette entremise, on est allé chercher une autre subvention, le CAP, ou le Community Animation Program, au fédéral. À partir de nos jardins communautaires, on a créé ce qu'on a appelé le Comité enviro-santé. Ça ressemblait à Villes et villages en santé du Québec ou aux coalitions-santé de l'Ontario. On voulait avoir une dimension environnementale et santé en même temps. Pour les fonctionnaires de la municipalité, le jardinage était un exemple, mais ils voulaient le faire selon une approche beaucoup plus globale. Nous nous sommes donc mis à travailler sur des aspects environnementaux, conjointement avec notre activité des jardins communautaires.

N.St-Amand : Donc, il y a un changement de focus?

M. Bisson : Tout à fait. Là on se tourne vers le global, vers l'environnement au sens global du terme. Les contacts avec ces fonctionnaires nous ont facilité l'accès à de la machinerie, à du travail qui pouvait se faire pour supporter les jardins communautaires aussi. Donc, on rejoignait nos deux intérêts, finalement.

À partir de l'été 1996, on a embauché une animatrice pour 4 mois, par l'entremise de ce programme d'animation fédéral. Là, nous avons créé, comme je vous disais, le Comité enviro-santé et nous étions 16 partenaires autour de la table : les Scouts, les gens de la municipalité, l'Institut de développement du St-Laurent, la Société d'horticulture de Cornwall. Nous sommes allés chercher plusieurs personnes s'intéressant à la problématique de l'environnement. Nous avions un petit terrain de jardinage dans le centre de la ville. Mais nous avions les yeux sur un autre terrain, tout près du quartier où logeaient les gens plus démunis, dans un secteur plus stratégique. Eux, dans leur vision globale, voulaient transformer ce terrain en parc écologique. Un nouveau jardin communautaire serait partie intégrante d'un parc écologique beaucoup plus vaste. Se retrouvaient là-dedans, entre autres, un milieu aquatique avec un étang, un jardin de papillons, une forêt 
communautaire, un jardin communautaire... donc, un projet beaucoup plus vaste, plus global. Avec la force du Comité envirosanté, on s'est mis à travailler sur ce projet-là. Et le petit 10000 \$ que nous allions chercher chaque année aidait à payer les factures. Nous sommes allés chercher aussi ce qu'on appelait à l'époque le Service jeunesse Canada, un autre programme du gouvernement fédéral. Cela nous donnait accès, pendant six mois, à des jeunes qui voulaient travailler à des projets particuliers. On a recruté quatre jeunes de Cornwall. Ils ont travaillé aux deux jardins communautaires. On en avait créé un deuxième à l'intérieur de l'Éco-parc—nom du parc écologique de Cornwall.Alors dès 1996, nous avions deux jardins communautaires...

N. St-Amand : Et le C.A.P. fournissait-il de l'argent?

M. Bisson : Oui, mais c'était très peu, ça. C'était une animatrice à temps partiel qui faisait la coordination du Comité enviro-santé. Disons que ce n'était pas de l'argent qu'on pouvait investir directement sur le terrain.Vu que nous avions les quatre jeunes travailleurs, nous avons investi notre $10000 \$ \ldots$ parce que le terrain écologique dont on parlait, il y avait rien là-dessus, c'était une terre de remplissage. Donc, j'ai pris l'argent pour acheter de la terre noire, puis on eu un partenariat avec la Domtar qui nous a fourni ce qu'on appelle du bio-solide. Nous avions ainsi un aspect "recherche environnementale » en réutilisant ça, mélangé avec de la terre noire. On avait un beau concept dans notre parc écologique.

Nous avons attiré beaucoup plus de jardiniers. Une vingtaine dès la deuxième année. Pendant ce temps-là, les jardins des écoles continuaient de fonctionner.

N.St-Amand : Toujours au même endroit ou dans les cours d'écoles?

M. Bisson : Le jardin communautaire fonctionnait toujours dans l'autre petit parc dont nous avons parlé et un autre dans la cour d'une école qui s'était montrée intéressée pour l'année suivante. Notre objectif allait bon train.

N.St-Amand : Vous aviez le vent dans les voiles, quoi!

M. Bisson : Nous avions le vent dans les voiles.Tellement que, l'année suivante, nous avons eu la visite de la Fondation Trillium, qui à l'époque octroyait le Prix d'entraide communautaire à l'échelle provinciale. 
On cherchait des candidats aux prix. Et la ville de Cornwall, par l'entremise de son parc écologique, donc de son jardin communautaire, à gagné le Prix d'entraide 1997 de la Fondation Trillium avec, en prime, une bourse de 20000 \$. Cet argent a été investi dans les jardins : cabanons, système éolien...

Nous avons ainsi eu la reconnaissance officielle que ce projet-là avait amené des choses intéressantes. Mais cela ne nous a pas arrêtés. Nous nous sommes rendus compte que les écoles étaient vraiment intéressées. Puis la venue de ces fonds-là libérait toujours les $25000 \$$ pour d'autres dépenses. Alors nous avons investi cette somme-là pour que les écoles puissent avoir leurs jardins à eux. Elles avaient certaines contraintes : ça leur prenait des clôtures, de la terre... Aujourd'hui, deux des quatre écoles impliquées au début ont maintenant des jardins communautaires et l'une d'elles s'est offert un programme de reverdissement de sa cour d'école. Les écoles sont maintenant autonomes; elles s'occupent de leurs affaires, elles n'ont plus besoin de nous. Des deux autres écoles à bénéficier du programme, une a fermé ses portes, dans la restructuration et l'autre est tellement grosse, il y a tellement de limites au niveau de son terrain, c'est vraiment l'école typique de centre-ville, avec de l'asphalte tout autour... Ça aurait été trop compliqué, mais ce n'était pas un manque d'intérêt. Deux des écoles étaient du secteur catholique, et deux du secteur public. Ces dernières ont été plus promptes à embarquer et à fonctionner. On aurait dit qu'il y avait plus de liberté, ou plus d'envie de faire les choses différemment des autres.

N.St-Amand : Les quatre écoles étaient francophones...

M. Bisson : Oui, deux catholiques puis deux publiques. Vous pourriez parler à l'une des directrices de ces deux écoles-là, elle vous parlera de l'enthousiasme soulevé. Et les professeurs aiment ça aussi—c'est vraiment intéressant!

Ça, c'est l'aspect " école ", mais il y a une autre dimension qui est intéressante : le Comité enviro-santé a élargi aussi les horizons dans notre secteur. Quand on fait du développement communautaire, c'est toujours ce que l'on souhaite. On ne veut pas juste semer des choses, on veut aussi qu'il y ait une récolte à un moment donné; on sème beaucoup de choses, on ne récolte pas tout le 
temps! Mais il y a des fois où l'on récolte. Entre autres, il y a des jardiniers qu'on cherchait à rejoindre. Ils sont venus dans nos jardins, en particulier dans celui de l'Éco-parc. À l'époque on avait deux jardins : le jardin Brookdale, près de la rue du même nom et le jardin Éco-parc, cultivé par des personnes habitant dans des logements à prix modique.

Une résidante de Glenn View Heights, un des endroits les plus démunis de Cornwall où se retrouvent de nombreux logements subventionnés, a importé le projet de jardin communautaire dans son quartier. Aujourd'hui, on y retrouve deux jardins qui fonctionnent de façon autonome. Tout ça parce que nous avons invité la directrice du quartier à notre Comité enviro-santé. Mais elle n'aurait jamais pu le faire s'il n'y avait pas eu, à l'intérieur de nos jardins, de ses propres locataires intéressés. Alors elle a facilité le travail des bénévoles du GlennView Heights, pour qu'ils puissent créer le jardin communautaire. Pour nous, c'était merveilleux. Des jardins communautaires dans ces secteurs particuliers ne pouvaient être une meilleure réponse à notre préoccupation initiale, l'insécurité alimentaire.

N. St-Amand : Dans les milieux comme tels, quoi!

M. Bisson : ...et en charge de leur milieu. Et ça, c'est l'un des aspects sur lesquels nous avons beaucoup travaillé. Je vous ai parlé des programmes de reverdissement. L'école publique Rose-des-vents a initié un tel projet. Mais je sais qu'avec d'autres membres du Comité enviro-santé, nous avons fait des présentations dans bien des écoles. Aujourd'hui, plusieurs s'offrent de tels programmes à Cornwall et dans STORMONT, DUNDAS et GLENGARRY.

N.St-Amand : ...qui ont un certain effet boule-de-neige.

M. Bisson : Un effet boule-de-neige à la suite de présentations de membres du Comité enviro-santé. Nous ne voulons pas prendre tout le crédit pour ce qui a été fait. Mais il y a eu quand même des présentations qui ont été faites, des intérêts qui ont été suscités, qui ont été stimulés par d'autres par la suite, et c'est tant mieux!

N.St-Amand : Quand vous parlez de programmes de reverdissement, parlezvous exclusivement de jardins, ou si vous parlez de compostage, de recyclage, de plantation d'arbres? 
M. Bisson : Souvent, en Ontario comme ailleurs au Canada, on a construit des cours d'écoles comme des stationnements. On faisait une cour en asphalte, puis érigeait une grosse clôture autour, puis on disait : «Les enfants vont s'amuser là ».

N.St-Amand : Ils vont être en sécurité.

M. Bisson : ...sauf que, lorsqu'on a une préoccupation environnementale, et qu'on suit tout ce qui a été fait dans d'autres pays, on se rend compte que les enfants peuvent jouer dans un environnement qui est beaucoup plus intéressant : il n'y a rien de mal à avoir de la pelouse, il n'y a rien de mal à avoir des arbres, il n'y a rien de mal à avoir ce qu'on appelle " des projets de naturalisation ", c'està-dire de laisser aller une zone particulière, laisser la nature suivre son cours... Évidemment on triche un peu : on sème certaines plantes et certaines fleurs, mais on laisse la nature aller décider de ce qu'elle va recréer comme milieu... Il y a plus d'une approche : on peut tout simplement planter un arbre dans une cour-ça va faire de l'ombre, ça va coûter beaucoup moins cher que de construire des structures qui vont amener l'ombre, et tout ça. Parler de reverdissement n'exclut pas le compostage, le recyclage...

N.St-Amand : Ça sensibilise en même temps.

M. Bisson : Exactement.Avec le Comité enviro-santé, nous sommes intervenus dans d'autres secteurs. Certains, par exemples, se sont intéressés aux pesticides. Et moi, encore là, l'oeil d'organisateur communautaire à l'affût de ce qui se passe, j'ai vu qu'à ce chapitre il y avait des gens qui n'étaient pas tout à fait satisfaits. Étant impliqués en environnement-santé, nous devions agir de façon très globale. Certains voulaient agir de façon plus précise, sur des thèmes comme l'usage des pesticides à des fins cosmétiques. Alors, nous, au Centre de santé, nous avons réuni quelques-uns de ces personnes avec un promoteur santé du Centre pour vider la question. De là est né un sous-comité qui s'appelle le Comité aux alternatives aux pesticides. Le C.A.P. comme on l'appelle aujourd'hui.

Ce comité est autonome par rapport au Comité enviro-santé, mais l'un de ses représentants y siège. Le C.A.P. a une fonction différente, beaucoup plus politique, plus éducative avec un rôle 
très précis de sensibilisation. Certains de ses bénévoles actuels ont travaillé à un projet de loi privé qui a été présenté au Parlement en vue de réglementer davantage l'usage des pesticides au Canada. D'autres ont fait circuler des pétitions, ont rencontré le député local... Vraiment, ils ont travaillé fort sur ce sujet.

N. St-Amand : Dans l'évolution du projet, est-ce qu'on parle toujours de gens défavorisés, ou si on parle de la population en général?

M. Bisson : Ça, c'est un autre côté de l'apport des jardins communautaires. Le comité aux alternatives aux pesticides, avec ses bénévoles, rejoint des gens de la classe moyenne avec des intérêts différents...

N.St-Amand : ...du genre, propriétaires de maisons, qui ont un petit parterre, etc?

M. Bisson : Exactement. Un autre développement s'est fait au Comité envirosanté avec une autre subvention stratégique de la fondation Canadian Living; nous sommes allés chercher un montant d'argent pour créer ce qu'on appelle l'Alliance de nutrition pour les enfants, parce que nous sommes toujours restés près des enfants. L'Alliance de nutrition pour les enfants regroupe le Bureau de santé de l'Est de l'Ontario, le Centre de santé communautaire de l'Estrie et encore une fois Partir d'un bon pas pour le volet scolaire. Elle s'est lancée avec la fondation Canadian Living dans un projet visant à distribuer des déjeuners et des collations de qualité aux enfants dans l'ensemble des écoles de S.D.G. Cette initiative semble éloignée des activités du Comité enviro-santé, mais elle vient quand même de celui-ci, d'où le lien avec le jardin communautaire initial...

N. St-Amand : ...des retombées inattendues, quoi!

M. Bisson : Exactement. Donc, à partir d'un projet de petit jardin communautaire, on peut parler de développement local orchestré, planifié.

N.St-Amand : Pourriez-vous dresser un portrait de la situation actuelle?

M. Bisson : On a une soixantaine de jardiniers, dont une quinzaine au petit jardin de la rue Brookdale, celui qui fonctionne de la façon la plus autonome. Les jardiniers l'ont pris en main. Ils s'occupent de l'entretien. L'organisateur du Centre de santé va faire un tour de temps en temps, mais c'est tout-ils sont vraiment autonomes. 
Le deuxième fait partie de l'Éco-parc. C'est le plus gros et c'est lui qui demande le plus de temps. Nous faisons souvent appel à des programmes comme Ontario au travail pour aider à la gestion de ce parc, parce qu'il est plus vaste qu'un simple jardin communautaire.

N.St-Amand : Et la main-d'oeuvre vous est fournie, à ce moment-là, gratuitement, par le programme?

M. Bisson : Oui, c'est ça. Certains travailleurs font de l'animation, d'autres travaillent la terre, facilitent le travail des jardiniers, s'occupent de l'entretien du cabanon, des outils, et arrosent quand les jardiniers ne sont pas là. Donc, ça fonctionne assez bien malgré quelques problèmes-la plupart des jardins communautaires, où qu'ils soient, vivent le même genre de difficultés, le vandalisme, le vol par exemple. On a eu des cas cocasses, comme celui des nouveaux arrivants à Cornwall qui ont entendu parler des jardins communautaires. Ils venaient simplement chercher des légumes dans le jardin : leur compréhension d'un jardin communautaire, c'était que c'était pour tout le monde. On s'est expliqué avec eux. C'était quand même assez drôle. Il y a aussi le problème de vandalisme, et ça, ça fait partie des enjeux. Malheureusement, nous avons dû mettre des clôtures autour des jardins, pour éviter ces problèmes. Ça, ce sont les aspects un peu moins intéressants... On a des jardiniers qui sont là depuis le début. Mais la plupart demeurent deux ou trois ans.

N. St-Amand : Ah bon! Puis après ça, qu'est-ce qui arrive?

M. Bisson : Après, ça dépend : soit que les gens décident de lâcher, tout simplement, ou ils trouvent que ça leur demande trop de temps; certains ont déménagé ou ont décidé de tenter l'expérience à leurs domiciles.

N.St-Amand : Donc, il y a un roulement, si on peut dire, des gens qui sont impliqués dans cette initiative. Ce qui crée un problème pour vous, au niveau d'une certaine stabilité, peut-être?

M. Bisson : Certaines personnes reviennent d'année en année. Mais il y a un aspect sur lequel nous devons travailler dans les prochaines années. Le terrain de l'Éco-parc, c'est un grand jardin communautaire; c'est gros, donc c'est plus difficile pour les gens de fraterniser. À 
Brookdale, il y a deux personnes qui prennent le jardin en charge. Parce que c'est plus petit, c'est plus faisable. Il se trouve dans un quartier précis, donc les gens peuvent se parler, se voir plus souvent. Parce que l'autre jardin est très vaste et situé plus au centre-ville, il y a des gens qui viennent d'un peu partout, de plus loin; les gens se connaissent moins, ce qui amène un autre problème, disons une difficulté supplémentaire, celle de réunir ces gens-là pour les corvées, alors qu'au petit jardin, c'est très facile. Prenons par exemple, le nettoyage de fin d'année : on a une très bonne participation dans le petit jardin; dans le grand jardin, il y en a qui se disent que quelqu'un va le faire.

N.St-Amand : ...les autres vont y aller.

M. Bisson : Exactement. Là, c'est un petit peu ça, c'est le défi disons plus «quotidien " qu'on a à rencontrer. Sinon, la demande est forte. Beaucoup de gens veulent participer aux jardins. Encore cette année, on a agrandi. On a ajouté une dizaine de lots; on pense bien que l'année prochaine, on aura à faire la même chose.

N. St-Amand : C'est un beau projet, c'est une belle histoire...

M. Bisson : Une excellente histoire, ah oui!

N.St-Amand : Quel est, selon vous, le rôle d'un animateur social, d'un " développeur " communautaire? Quel est votre rôle là-dedans?

M. Bisson : Moi, c'est vraiment le rôle de facilitateur. Premièrement, il y a eu la question de faire la demande de subvention, d'aller la chercher, de réunir les bonnes personnes et de préciser des objectifs clairs. On savait qu'on voulait intervenir au niveau des écoles, mais notre premier objectif visait les personnes démunies qui avaient identifié les jardins communautaires comme étant un besoin. Maintenir le cap sur ces objectifs-là, c'est bien important. Puis faciliter le plus possible : les écoles ont des contraintes, ce n'est pas facile de faire bouger des écoles! Ils ont des curriculums compliqués, les tâches des enseignants sont de plus en plus lourdes. Donc, il faut arriver, le plus possible, à leur faciliter la tâche. Lorsqu'on leur a présenté le projet de jardins communautaires, on est arrivé avec une animatrice, donc avec de l'aide. L'enseignant n'était responsable que d'amener son groupe d'élèves. Tout l'aspect logistique, nous $\mathrm{y}$ voyions nous-mêmes. 
Il faut de la diplomatie aussi. Cela n'a pas été facile au début; nous aurions pu nous décourager. Avec la municipalité en particulier. Mais on a appris que, quand on croit à un projet, qu'on sait qu'il $\mathrm{y}$ a des personnes qui y croient aussi, il faut persévérer — c'est très important. Et il faut impliquer, le plus possible, la clientèle qu'on cherche à rejoindre. Dès qu'on a eu quelques personnes intéressées par ce projet-là-des bénévoles et des jardiniers—dès qu'on a eu ces gens-là, ça été beaucoup plus facile. Parce que ce sont nos meilleurs vendeurs. Ce sont vraiment eux qui sont capables d'aller rejoindre les personnes de leur entourage. Il faut agir comme facilitateur auprès d'eux autres aussi : il faut les comprendre, respecter ce qu'ils demandent, leur rythme de travail aussi-ce n'est pas toujours évident.

N. St-Amand : Dernière question, peut-être... Les enfants, là-dedans? Parce que c'est, comme l'expression le dit, «les enfants d'abord ». Ce sont les enfants, les enfants pauvres en particulier, que vous vouliez aller chercher. Est-ce que votre expérience est concluante, dans le sens que les enfants sont maintenant plus sensibilisés, plus engagés? C'est à travers les enfants-je pense que c'était un de vos objectifs-que vous allez modifier un peu l'insécurité alimentaire dans le milieu?

M. Bisson : Deux écoles publiques—je sais que ces écoles-là rejoignent des clientèles assez défavorisées — ont fait du jardinage communautaire dans leur propre cour. Les deux ont fait du reverdissement. Elles sont donc sensibilisées à l'environnement et participent à l'Alliance de nutrition des enfants. Je pense que la graine est là. Les étés où des enfants étaient impliqués, les deux étés où, vraiment, les écoles y allaient, j'ai vu, de mes propres yeux, des enfants aller montrer à leurs parents ce qu'ils avaient semé. Ça, ça en dit beaucoup! J'ai eu des témoignages de gens qui ont vu plusieurs enfants aller montrer ça à la famille, même à des grands-parents. Des enfants amenaient leurs grands-parents voir ce qu'ils avaient fait pousseril y a tout un sentiment, il y a tout un message qui est donné par l'enfant. Tout a été planifié selon des bases écologiques, aussi : on n'utilisait jamais de produits chimiques. C'était très important. C'est l'aspect que le Centre de santé a toujours voulu défendre... Partir d'un bon pas aussi, qui fait de la prévention primaire. On 
avait donc une dimension écologique : respecter l'équilibre, respecter les difficultés de certaines plantes. On ne réussit pas toujours nos semailles. Il faut laisser la nature fonctionner là-dedans. Puis ça aussi, c'est une leçon que l'enseignant peut récupérer.

N.St-Amand : Un mot de la fin, peut-être?

M. Bisson : Le mot de la fin, c'est que je pense que les programmes des jardins communautaires sont facilement exportables partout. Je suis convaincu qu'il y a moyen de les faire fonctionner. Il y a d'autres exemples en province aussi. Je sais qu'au Québec, dans la région de Montréal, ça fonctionne très fort. Moi,je suis allé en visiter, les premières années. Ça nous a encouragés. Un aspect intéressant pour nous a été la réunion des francophones et des anglophones : en cours de jardinage, il n'y a pas de langue...

N. St-Amand : ...c'est la langue de la terre.

M. Bisson : Oui. Au début, nos enfants venaient des écoles françaises. Par la suite, d'autres se sont ajoutés au point qu'à Cornwall, ce qui est assez rare, nous avons un jardin multi-ethnique, oui à Cornwall, une ville qui est quand même reconnue comme étant assez "blanche ", anglo-saxonne et francophone. Nous avions cet échange culturel avec des Haïtiens, des Amérindiens, des Indiens... à l'intérieur des jardins. Cela apportait un aspect intéressant, ça aussi.

N. St-Amand : Ça diversifie la culture, à toutes sortes de niveaux.

M. Bisson : Absolument. On a eu des réunions de fin d'année, les réunions d'évaluation, où des membres des diverses communautés apportaient des plats préparés à partir de ce qu'ils avaient récolté dans le jardin. Ils offraient à d'autres jardiniers, typiquement francoontariens, ou typiquement anglo-saxons, de la nourriture plus épicée...c'était bien intéressant! 